\title{
Ramucirumab: preclinical research and clinical development
}

This article was published in the following Dove Press journal:

OncoTargets and Therapy

29 October 2014

Number of times this article has been viewed

\author{
Giuseppe Aprile' \\ Erika Rijavec $^{2}$ \\ Caterina Fontanella' \\ Karim Rihawi' \\ Francesco Grossi ${ }^{2}$ \\ 'Department of Oncology, University \\ Hospital of Udine, Udine, Italy; \\ ${ }^{2}$ Lung Cancer Unit, National Cancer \\ Institut "San Martino", Genoa, Italy
}

Correspondence: Giuseppe Aprile

Department of Oncology, University and General Hospital, Piazzale S Maria della

Misericordia 15, 33 I 00 Udine, Italy

Tel +39432559308

Fax +39 432559305

Email aprile.giuseppe@aoud.sanita.fvg.it
Abstract: Ramucirumab (IMC-1121B, LY3009806), a fully humanized monoclonal antibody directed against the extracellular domain of vascular endothelial growth factor receptor 2 (VEGFR-2), is a new therapeutic option that selectively inhibits the human VEGFR-2 with a much greater affinity than its natural ligands. Based on the promising results of both preclinical and early clinical studies, ramucirumab has been tested in different tumor types either alone or in combination with chemotherapy. While it has recently been granted its first US Food and Drug Administration approval for use as a single agent in patients with advanced or metastatic gastric cancer or gastroesophageal junction carcinoma, its role for metastatic breast cancer or advanced non-small-cell lung cancer is still debated. The aims of this review are to recall and discuss the most significant preclinical and clinical studies that led to the development of ramucirumab and to present the results of the randomized clinical trials that have tested its efficacy in different malignancies, including gastric and lung cancer.

Keywords: ramucirumab, gastric cancer, lung cancer, breast cancer, antiangiogenic

\section{Introduction}

Soon after the central role of the vascular endothelial growth factor (VEGF) pathway in cancer growth was established, ${ }^{1}$ the widespread clinical use of angiogenic inhibitors led to significant advances in different cancers. ${ }^{2}$ The notable survival effect of these drugs, however, does not significantly impact on the overall cure rate; moreover, the average benefit rapidly decreases with time because of the existence of redundant pathways or the early emergence of resistance mechanisms. ${ }^{3}$ Notably, despite the massive efforts made to identify potential predictive factors that might help select patients who are more likely to benefit from these drugs, all the results achieved so far are disappointing. ${ }^{4,5}$ Additionally, concerns regarding the benefit-to-risk ratio of these drugs may cause embitterment and limit their use in specific populations. ${ }^{6}$ VEGF receptor 2 (VEGFR-2), a type II transmembrane tyrosine kinase receptor expressed on endothelial cells and on circulating bone marrow-derived endothelial progenitors cells, is standing out as a key receptor in tumor neoangiogenesis. ${ }^{7}$ This complex molecule contains three different parts (including an immunoglobulin [Ig]-like domain extracellular region, a hydrophobic transmembrane region containing the tyrosine kinase domain, and the carboxyl terminal tail) and binds all VEGF-A isoforms, VEGF-C, and VEGF-D. Its unique three-dimensional structure has consequently spurred the research to develop novel specific targeted inhibitors. ${ }^{8}$ Ramucirumab (IMC-1121B, LY3009806) is a fully humanized monoclonal antibody directed against the extracellular domain of VEGFR-2 that can be safely administered intravenously every 2 or 3 weeks. ${ }^{9}$ Preclinical models showed that ramucirumab might 
selectively bind to and inhibit the human VEGFR-2 ${ }^{10}$ with a much greater affinity than its natural ligands ${ }^{11}$ (see Figure 1). Once the early clinical studies were completed showing satisfactory results, this interesting molecule was further investigated within a planned program of Phase III registration trials, given either alone or combined with chemotherapy. Recently, ramucirumab has received its first US Food and Drug Administration approval for use as monotherapy in patients with advanced or metastatic gastric cancer or gastroesophageal junction (GEJ) carcinoma who had previously failed first-line chemotherapy. ${ }^{12}$ The aims of this short review are to recall and comment on the most significant preclinical and clinical steps that have witnessed the development of ramucirumab and to present the results of the randomized studies that have tested its efficacy in different malignancies, including gastric carcinomas and lung cancers (see Table 1).

\section{The biology behind ramucirumab and early clinical trials}

VEGFs are a family of glycoproteins that play a critical role and regulate vascular development, angiogenesis,

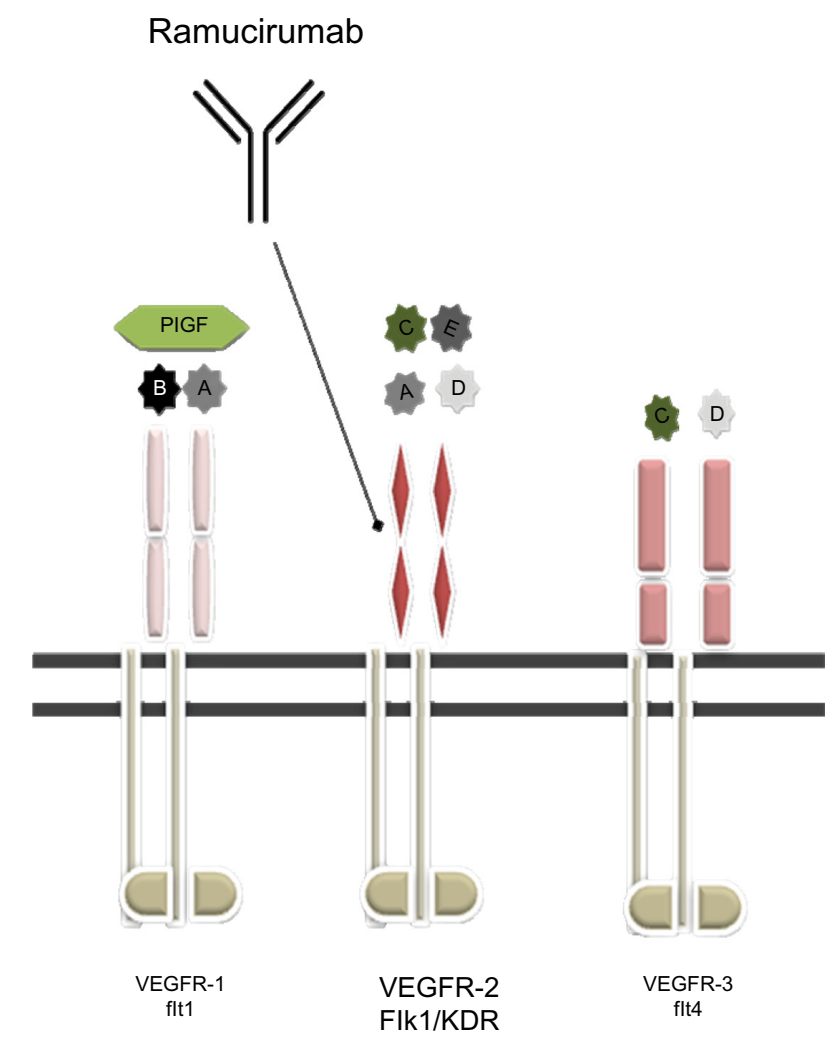

Figure I A graphical representation of the effect of ramucirumab when blocking VEGFR-2. VEGF ligands and their receptors are represented. A: VEGF-A, B: VEGF-B, C: VEGF-C, D: VEGF-D, E: VEGF-E.

Abbreviations: VEGFR-2, vascular endothelial growth factor receptor 2; VEGF, vascular endothelial growth factor; PIGF, placental growth factor. and lymphoangiogenesis. Five different VEGF ligands bind in a specific manner to three different, although structurally related, receptor tyrosine kinases: VEGFR-1, mainly involved in hematopoietic cell development; VEGFR-2, critical for vascular endothelial cell development; and VEGFR-3, critical for lymphatic endothelial cell development. The role of VEGFR-2 was initially shown to be fundamental in vascular development by the fact that VEGFR-2 ${ }^{-/}$knockout mice died due to defective development of blood islands, endothelial cells, and hematopoietic cells. Various studies have also shown that VEGFR-2, the target of ramucirumab, is the main mediator of several physiological and pathological effects of VEGF-A on endothelial cells. These include proliferation, migration, survival, and permeability. In adults, VEGFR-2 is expressed mainly on vascular endothelial cells, megakaryocytes, and hematopoietic stem cells. The importance of VEGFR-2 in tumor angiogenesis suggests that blockade of this receptor would be a useful therapeutic strategy for inhibiting angiogenesis and tumor growth. Based on these premises, ramucirumab was developed. Both ramucirumab and its murine version, DC-101, were designed to prevent VEGFR-2 activation, resulting in significant antitumor activity in a wide range of malignancies in animal models. ${ }^{13-15}$ One of the first highly selective inhibitors of VEGFR-2 was actually DC-101, which showed to directly inhibit angiogenesis as well as the growth of several mouse and human tumors in in vivo models, ${ }^{13}$ providing evidence that the blockade of this pathway is a useful strategy for the treatment of human cancer. However, one of the major obstacles in developing antibody-based therapeutics was the immunogenicity of the nonhuman-derived monoclonal antibodies. This spurred the production of ramucirumab (IMC-1121B; ImClone Systems, New York, NY, USA), a fully human IgG1 monoclonal antibody, which was shown to be less immunogenic than antibodies of nonhuman origin and therefore better tolerated in human therapy. ${ }^{16}$

The Phase I trial was conducted in order to establish the safety, tolerability, and maximum tolerated dose (MTD) of ramucirumab in patients with advanced solid tumors refractory to standard treatment. ${ }^{17}$ The dose escalation phase included 37 patients who were treated across seven different dose levels. The initial dose level of $2 \mathrm{mg} / \mathrm{kg}$, based on the results of pharmacokinetic (PK) and preclinical data, was subsequently increased by $100 \%$ (4 mg/kg), $50 \%(6 \mathrm{mg} / \mathrm{kg}), 33 \%$ (8 mg/kg), and 25\% (10 mg/kg, $13 \mathrm{mg} / \mathrm{kg}$, and $16 \mathrm{mg} / \mathrm{kg})$. Intrapatient dose escalation was not allowed. Ramucirumab was administered at escalating doses as a 1-hour intravenous infusion during cycles consisting of four weekly infusions 
Table I A summary of the Phase III trials

\begin{tabular}{|c|c|c|c|c|c|}
\hline Trial & Study population & Study design & $\begin{array}{l}\text { Primary } \\
\text { end point }\end{array}$ & $\begin{array}{l}\text { Results } \\
\text { (in months) }\end{array}$ & $P$-value \\
\hline $\begin{array}{l}\text { NCT00917384 } \\
\text { REGARD }\end{array}$ & $\begin{array}{l}\text { MG or MGEJ progressed during } \\
\text { or following first-line platinum- or } \\
\text { fluoropyrimidine-based therapy }\end{array}$ & $\begin{array}{l}\text { Ramucirumab } 8 \mathrm{mg} / \mathrm{kg} \text { every } 14 \text { days vs } \\
\text { placebo }\end{array}$ & OS & 5.2 vs 3.8 & 0.0473 \\
\hline $\begin{array}{l}\text { NCTOII } 70663 \\
\text { RAINBOW }\end{array}$ & $\begin{array}{l}\text { MG progressed during or following } \\
\text { first-line platinum- or fluoropyrimidine- } \\
\text { based therapy }\end{array}$ & $\begin{array}{l}\text { Paclitaxel } 80 \mathrm{mg} / \mathrm{m}^{2}+\text { ramucirumab } \\
8 \mathrm{mg} / \mathrm{kg} \text { every } 14 \text { days vs paclitaxel } \\
80 \mathrm{mg} / \mathrm{m}^{2}+\text { placebo every } 14 \text { days }\end{array}$ & OS & 9.6 vs 7.4 & 0.0169 \\
\hline $\begin{array}{l}\text { NCTOII } 68973 \\
\text { REVEL }\end{array}$ & $\begin{array}{l}\text { MNSCLC progressed during or } \\
\text { following first-line platinum-based } \\
\text { therapy }\end{array}$ & $\begin{array}{l}\text { Docetaxel } 75 \mathrm{mg} / \mathrm{m}^{2}+\text { ramucirumab } \\
10 \mathrm{mg} / \mathrm{kg} \text { every } 21 \text { days vs docetaxel } \\
75 \mathrm{mg} / \mathrm{m}^{2}+\text { placebo every } 21 \text { days }\end{array}$ & OS & 10.5 vs 9.1 & 0.02 \\
\hline $\begin{array}{l}\text { NCT00703326 } \\
\text { ROSE/TRIO-I2 }\end{array}$ & $\begin{array}{l}\text { Previously untreated locally advanced } \\
\text { or MBC HER2-negative }\end{array}$ & $\begin{array}{l}\text { Docetaxel } 75 \mathrm{mg} / \mathrm{m}^{2}+\text { ramucirumab } \\
10 \mathrm{mg} / \mathrm{kg} \text { every } 21 \text { days vs docetaxel } \\
75 \mathrm{mg} / \mathrm{m}^{2}+\text { placebo every } 21 \text { days }\end{array}$ & PFS & 9.5 vs 8.2 & 0.077 \\
\hline $\begin{array}{l}\text { NCTOII } 83780 \\
\text { RAISE }\end{array}$ & $\begin{array}{l}\text { MCRC progressed during or following } \\
\text { first-line therapy with bevacizumab, } \\
\text { oxaliplatin, and fluoropyrimidine }\end{array}$ & $\begin{array}{l}\text { FOLFIRI + ramucirumab } 8 \mathrm{mg} / \mathrm{kg} \text { every } \\
\text { I4 days vs FOLFIRI }\end{array}$ & OS & Trial ongoing & - \\
\hline $\begin{array}{l}\text { NCTOI I } 40347 \\
\text { REACH }\end{array}$ & $\begin{array}{l}\text { HCC progressed during or following } \\
\text { sorafenib and with Child-Pugh } \\
\text { score of } A\end{array}$ & $\begin{array}{l}\text { Ramucirumab } 8 \mathrm{mg} / \mathrm{kg} \text { every } 14 \text { days vs } \\
\text { placebo }\end{array}$ & OS & Trial ongoing & - \\
\hline
\end{tabular}

Abbreviations: NCT, number of clinical trial; MG, metastatic gastric adenocarcinoma; MGEJ, metastatic gastroesophageal junction adenocarcinoma; OS, overall survival; MNSCLC, metastatic non-small-cell lung cancer; MBC, metastatic breast cancer; HER2, human epidermal growth factor receptor 2; PFS, progression-free survival; MCRC, metastatic colorectal cancer; FOLFIRI, irinotecan $180 \mathrm{mg} / \mathrm{m}^{2}$ day I + 5-fluorouracil $2.4 \mathrm{mg} / \mathrm{m}^{2}$ days I-2 + folinic acid $400 \mathrm{mg} / \mathrm{m}^{2}$ day I; HCC, hepatocellular carcinoma.

followed in the first cycle by a 2-week rest for PK sampling; this treatment-free period was subsequently eliminated in an amendment. Patients who had received prior treatment with VEGF or VEGFR inhibitors or any monoclonal antibody, as well as patients with poor functional status, as determined by an Eastern Cooperative Oncology Group (ECOG) performance status $>1$, were excluded from the study. No doselimiting toxicities (DLTs) were observed at doses $<10 \mathrm{mg}$ / $\mathrm{kg}$. Overall, two patients experienced DLTs, with one patient developing a grade 3 hypertension after receiving the fourth dose of ramucirumab (dose level $10 \mathrm{mg} / \mathrm{kg}$ ) and another patient who had a DVT at a $16 \mathrm{mg} / \mathrm{kg}$ dose level. In this cohort, a third patient developed a grade 3 hypertension after the first dose of cycle 2, but this event, by definition, was not deemed a DLT. However, due to its temporary proximity to cycle 1, the MTD of ramucirumab was finally established at $13 \mathrm{mg} / \mathrm{kg}$ on a weekly schedule.

Overall, the toxicity profile of ramucirumab was similar to the one observed with other agents targeting the VEGFR-2/ VEGF axis. As a matter of fact, the principal toxicities reported were hypertension, vascular thrombotic events, and proteinuria. Hypertension generally resolved after administration of antihypertensive drugs, enabling patients to stay on trial, or after treatment suspension, with only one patient who had to permanently discontinue the drug. The most frequently described adverse events (AEs), involving $\geq 25 \%$ of all patients, regardless of relation to ramucirumab, were fatigue $(51.4 \%)$, headache $(51.4 \%)$, peripheral edema (35.1\%), diarrhea (35.1\%), nausea (32.4\%), upper respiratory tract infection $(32.4 \%)$, abdominal pain $(29.7 \%)$, anorexia (29.7\%), constipation (29.7\%), epistaxis (29.7\%), proteinuria $(29.7 \%)$, arthralgia $(27.0 \%)$, cough $(27.0 \%)$, and dyspnea $(27.0 \%)$.

The Phase I trial not only showed that ramucirumab was well tolerated as a weekly 1-hour infusion but also provided proof that VEGFR-2 blockade with such a monoclonal antibody could be an effective anticancer strategy. As a matter of fact, four patients (11\%) had confirmed partial response (PR), including two patients with melanoma and gastric cancer treated at a $4 \mathrm{mg} / \mathrm{kg}$ dose level, one patient with uterine leiomyosarcoma enrolled in the $13 \mathrm{mg} / \mathrm{kg}$ cohort, and one patient with ovarian carcinoma who received ramucirumab at a $16 \mathrm{mg} / \mathrm{kg}$ dose level. Furthermore, eleven patients (30\%) with different types of tumor experienced either PR or SD lasting $\geq 6$ months.

Despite the MTD being established at $13 \mathrm{mg} / \mathrm{kg}$, the dose eventually selected for subsequent trials was $8 \mathrm{mg} / \mathrm{kg}$, based on PK results. With a clearance mechanism likely to be receptor mediated, ramucirumab PK clearance is saturated at $8 \mathrm{mg} / \mathrm{kg}$, leading to the hypothesis that all VEGFR-2 are blocked by the drug at this dose level.

Finally, another interesting aspect observed in this trial was the relationship between ramucirumab and VEGF-A ligand. Because ramucirumab's affinity for VEGFR-2 is 
much higher than VEGF-A ligand, an increase of the serum concentration of the latter was expected as a result of its displacement from the receptor. VEGF-A concentrations actually increased 1.5 - to 3.5-fold across dose levels, mainly evident at doses $\geq 8 \mathrm{mg} / \mathrm{kg}$, and VEGF-A elevation after treatment with ramucirumab might be considered for estimating the adequacy and extent of VEGFR-2 blockade.

\section{Gastrointestinal cancers: the road to first global approval and beyond}

REGARD is an international, randomized, placebo-controlled, Phase III trial that was conducted between October 2009 and January 2012 in 29 countries all over the world. ${ }^{18}$ Patients with advanced or metastatic gastric cancer or GEJ carcinoma who had previously failed a first-line platinumbased chemotherapy were randomly assigned in a $2: 1$ ratio to intravenous ramucirumab $8 \mathrm{mg} / \mathrm{kg}$ once every 2 weeks $(n=238)$ or placebo $(n=117)$, in addition to best supportive care. The primary end point of the study was overall survival (OS); secondary end points included progression-free survival (PFS) and quality of life (QoL). Importantly, blinding involved the study sponsor, investigators, and enrolled patients. Randomization was stratified according to weight loss, anatomic location of the primary tumor, and geographic region. The final results were published after 278 patients had died. Compared with placebo, ramucirumab combined with best supportive care significantly prolonged median OS from 3.8 months to 5.2 months, translating into a $22 \%$ reduction in the risk of death (hazard ratio [HR] 0.77; 95\% confidence interval [CI] 0.60-0.99; $P=0.0473$ ). The study also met its secondary end points. Patients enrolled in the ramucirumab arm had an improvement in PFS (HR 0.48; 95\% CI $0.37-0.62 ; P<0.0001)$ and a significant increase in DCR (48.7\% vs 23.1\% with placebo; $P<0.0001$ ), although the overall risk reduction (RR) was equally low in both treatment arms (3.4\% vs 2.6\%). Subgroup analysis showed a consistent treatment effect across subgroups. Interestingly, male patients exposed to ramucirumab seemed to have a greater survival benefit compared with female patients (HR for OS 0.67 ; 95\% CI $0.49-0.91$ vs HR 1.43 ; 95\% CI $0.85-2.40)$. Although there was no age restriction among the inclusion criteria, median age of the enrolled patients was low in both arms (60 years). The antiangiogenic drug was very well tolerated, and no unexpected toxicity was reported. In the REGARD trial, $57 \%$ of the patients exposed to ramucirumab had grade 3-4 AEs compared with 58\% in the placebo group. The most frequent treatment-related severe $\mathrm{AE}$ was hypertension ( $8 \%$ with ramucirumab vs $3 \%$ with placebo); no grade 4 hypertension was recorded. Ramucirumab was not associated with increased rates of proteinuria, bleeding, venous thrombosis, or gastrointestinal perforation. In both study arms, $2 \%$ of the deaths were considered to be treatment related. The QoL assessment analysis showed that 34\% of patients in the ramucirumab arm reported stable or improved conditions at the 6-week assessment compared with $13 \%$ in the placebo group. One hundred and twenty-eight patients aged $\geq 65$ years were enrolled in the trial. A treatment by age subgroup interaction analysis showed that older patients exposed to ramucirumab had similar survival improvements compared with younger patients (age $>65$ years: HR 0.72 ; 95\% CI $0.47-1.1$ vs age $<65$ years: HR $0.84 ; 95 \%$ CI $0.61-1.17 ; P$ for interaction $=0.56) .{ }^{19}$ Similar results were reported for PFS. Notably, safety and tolerability profiles were also comparable between the two age cohorts.

The core results of RAINBOW, a large Phase III trial testing ramucirumab in pretreated patients with advanced or metastatic gastric or GEJ adenocarcinoma, have also been reported. ${ }^{20}$ The study randomized (1:1 ratio) 665 patients who had progressed while on or within 4 months of standard first-line treatment with a platinum-based chemotherapy to paclitaxel $80 \mathrm{mg} / \mathrm{m}^{2}$ alone or in combination with ramucirumab $8 \mathrm{mg} / \mathrm{kg}$ given every 2 weeks. OS was the primary study end point. Stratification factors included geographic region, measurable versus nonmeasurable disease according to Response Evaluation Criteria in Solid Tumors, and time to progression on first-line therapy ( $<6$ months vs $>6$ months). Within the whole trial population, 398 patients were from Europe, Australia, or North America (defined as region 1), 44 were from South America (region 2), and 223 from East Asia (region 3). The trial met its primary and secondary end points with a $19 \%$ reduction in the risk of death $(P=0.0169)$ and a $27 \%$ reduction in the risk of disease progression $(P<0.0001)$ with the addition of ramucirumab to paclitaxel. Median OS was 9.6 months for the combination versus 7.4 months for paclitaxel alone, and median PFS was 4.4 months versus 2.9 months, respectively. In addition, the DCR was $80 \%$ with paclitaxel plus ramucirumab versus $64 \%$ with paclitaxel alone $(P<0.0001)$. A similar proportion of patients received at least one postdiscontinuation treatment: $47.9 \%$ in the ramucirumab plus paclitaxel arm versus $45.4 \%$ in the paclitaxel alone arm. Overall, grade 3-4 AEs significantly increased with the addition of ramucirumab to paclitaxel ( $82 \%$ vs $63 \%$ ), raising safety concerns for the combination. More specifically, a higher incidence of grade 3-4 neutropenic events (40.7\% vs $18.8 \%$ ), leukopenia ( $17.4 \%$ vs $6.7 \%$ ), and hypertension ( $14.1 \%$ vs $2.4 \%$ ) was noted in the 
experimental arm. This increased frequency of AEs, however, did not lead to a higher rate of treatment discontinuation. Accordingly, the incidence of treatment-related deaths was similar across treatment arms ( $4.0 \%$ vs $4.6 \%)$. The authors used a prespecified stepwise Cox regression analysis to select baseline covariates associated with survival in the overall population, and then adjusted it for these factors in a Cox proportional hazards model that included a term for treatment arm. ${ }^{21}$ The stepwise Cox model identified seven independent survival predictors: Asian origin (region 3), ECOG PS 0, weight loss $<10 \%$ in the previous 3 months, a limited number of metastatic sites, absence of ascites, well-differentiated tumor histology, and prior gastrectomy. After adjusting for these potential prognostic factors in a multivariate analysis, OS benefit improved in the overall population and in the majority of subgroups, reinforcing the robustness of the primary analysis results. A separate analysis was conducted on region 1 patients. ${ }^{22}$ Compared with the overall population, patients from Europe, Australia, or North America were more likely to receive a triplets in first-line (37.2\% vs $24.5 \%$ ), to be diagnosed with a GEJ location of the primary tumor ( $30.7 \%$ vs $20.6 \%$ ), and to have widespread disease (metastasis in at least three different anatomic sites: $39.9 \%$ vs $33.7 \%$ ). The comparative analysis demonstrated that efficacy and safety results achieved among patients from Western countries were consistent with those of the overall study population. A QoL analysis, which was the secondary end point of the study, was also performed, showing that the addition of ramucirumab to paclitaxel did not impair the QoL, and that patients exposed to the experimental drug had a longer period of well-being and higher rates of stable or improved QLQ C-30 scores. ${ }^{23}$

The combination of ramucirumab and chemotherapy as first-line treatment did not return similar benefits. In a randomized, placebo-controlled, Phase II trial, 164 patients with previously untreated advanced esophageal, GEJ, or gastric cancers received oxaliplatin, leucovorin, and 5-fluorouracil (modified FOLFOX) plus ramucirumab $8 \mathrm{mg} / \mathrm{kg}$ or modified FOLFOX plus placebo. ${ }^{24}$ PFS was the primary end point of the study, while secondary end points included overall response rate (ORR), safety, and OS. Although a higher DCR was reported for patients receiving ramucirumab $(85 \%$ vs $67 \%$ ), in the intention-to-treat analysis, no difference in either median PFS (6.44 months vs 6.74 months; HR 0.98; 95\% CI $0.69-1.37)$ or median OS (11.7 months vs 11.5 months; HR 1.08; 95\% CI 0.73-1.58) was noted between treatment arms. Interestingly, reasons for treatment discontinuation other than disease progression were numerically higher in the experimental arm (48\% vs 16\%). As suggested by an exploratory analysis that showed longer PFS favoring ramucirumab in the gastric/GEJ subgroup, differences in baseline disease biology and dissimilar treatment sensibility to angiogenic inhibitors between esophageal and gastric cancers might have jeopardized the results of the trial.

$\mathrm{REACH}^{25}$ and RAISE ${ }^{26}$ are two large, second-line, randomized Phase III trials testing ramucirumab in pretreated patients with hepatocellular carcinoma or advanced colorectal carcinoma, respectively. The design of both trials has been presented at the 2012 American Society of Clinical Oncology (ASCO) Annual Meeting. ${ }^{27,28}$ In REACH, 544 hepatocellular carcinoma patients whose disease progressed during or following first-line therapy with sorafenib or who were intolerant to the agent were randomized to either ramucirumab or placebo. The trial enrollment has recently been completed, and final results of the study are expected in 2015. In the meantime, a press notice released on June 11, 2014 announced that OS favored the ramucirumab arm, but the advantage was not statistically significant. In the RAISE trial, over 1,000 advanced colorectal carcinoma patients who have failed a first-line combination including bevacizumab, oxaliplatin, and a fluoropyrimidine are being randomized to irinotecan, leucovorin, and 5-fluorouracil (FOLFIRI) plus ramucirumab versus FOLFIRI alone.

\section{Ramucirumab in lung cancer: renewed hope from antiangiogenic treatments}

In a first-line Phase II study enrolling advanced non-small-cell lung cancer (NSCLC) patients, Camidge et $\mathrm{al}^{29}$ investigated the combination of ramucirumab $(10 \mathrm{mg} / \mathrm{kg})$, carboplatin (AUC 6), and paclitaxel $\left(200 \mathrm{mg} / \mathrm{m}^{2}\right)$ every 3 weeks for a maximum of six cycles, followed by maintenance with ramucirumab alone. PFS at 6 months was the primary end point of the study. Fifteen of the first 30 patients enrolled were evaluable for response. Objective response rate was $67 \%$ (nine patients achieved a PR and one patient a complete response). Preliminary PFS at 6 months was 5.7 months. Preliminary safety data showed that the combination was well tolerated. At the 2013 World Conference on Lung Cancer, Doebele et al ${ }^{30}$ presented the results of a randomized Phase II study evaluating the administration of ramucirumab in combination with first-line platinum-based chemotherapy in 140 patients with nonsquamous advanced NSCLC. Patients were randomized in a 1:1 ratio to receive a maximum of six cycles of either cisplatin or carboplatin and pemetrexed every 3 weeks followed by maintenance treatment with pemetrexed ( $\operatorname{arm} A)$ or 
ramucirumab (at the dose of $10 \mathrm{mg} / \mathrm{kg}$ ) in combination with either cisplatin or carboplatin and pemetrexed every 3 weeks followed by maintenance treatment with ramucirumab and pemetrexed (arm B). PFS was the primary end point of the study. There was no significant difference in PFS between the two arms: 5.6 months in arm A versus 7.2 months in arm B (HR 0.75; $P=0.132$ ). A significant difference in terms of DCR, however, was observed: $86 \%$ for patients treated with chemotherapy and ramucirumab and $70 \%$ for patients treated with chemotherapy alone $(P=0.031)$. The addition of ramucirumab to chemotherapy did not lead to increased or unexpected toxicities. The most common grade $\geq 3$ AEs reported were neutropenia, thrombocytopenia, nausea, hypertension, back pain, and fatigue.

The combination of ramucirumab $(10 \mathrm{mg} / \mathrm{kg})$ and docetaxel $\left(75 \mathrm{mg} / \mathrm{m}^{2}\right)$, compared with docetaxel $\left(75 \mathrm{mg} / \mathrm{m}^{2}\right)$ plus placebo, has also been evaluated in a randomized, double-blind, Phase III study (REVEL study) recruiting advanced NSCLC patients who had experienced disease progression during or after previous first-line platinum-based chemotherapy. ${ }^{31}$ The treatment was administered until disease progression, unacceptable toxicity, or death. OS was the primary end point of the study. Secondary end points included PFS, ORR, patient-reported outcomes, and assessment of safety and toxicity. Exploratory objectives included the assessment of potential predictive biomarkers for response to ramucirumab from patients' sera and tumor specimens. The results of this pivotal trial have been recently published. ${ }^{32}$ The patients enrolled in the experimental arm $(n=628)$ achieved a significantly longer OS compared with those enrolled in the control arm $(n=625): 10.4$ versus 9.1 months (HR 0.857; $P=0.0235$ ). These results were observed in both squamous and nonsquamous patients. Patients treated with ramucirumab and docetaxel had a longer PFS (4.5 vs 3.0 months; HR 0.762; $P<0.0001)$ and a higher ORR (22.9\% vs $13.6 \%$; $P<0.001)$ compared with patients treated with docetaxel alone. The most frequently observed severe AEs in the experimental arm were fatigue (14\% vs $10 \%)$, neutropenia (49\% vs 40\%), leukopenia ( $14 \%$ vs 12\%), febrile neutropenia ( $16 \%$ vs $10 \%$ ), and hypertension ( $6 \%$ vs $2 \%$ ). Overall, the toxicities observed with ramucirumab were easily manageable with dose adjustments or supportive care.

Currently, a randomized Phase II study evaluating the role of platinum-based chemotherapy with or without ramucirumab as first-line treatment for patients with recurrent or advanced NSCLC is active, but it is not recruiting patients. ${ }^{33}$ Another randomized, double-blind, Phase II study comparing the combination of ramucirumab and docetaxel with docetaxel alone in Japanese patients who have experienced disease progression after a first-line platinum-based chemotherapy is currently ongoing. ${ }^{34}$

\section{Ramucirumab in metastatic breast cancer: a stinging setback}

The rationale for the use of an angiogenesis inhibitor in patients with metastatic breast cancer (MBC) derives from extensive laboratory data. In preclinical models, transfection of tumor cells with angiogenic stimulatory peptides has shown to increase tumor growth and to promote invasion and metastasis development. ${ }^{35}$ In vivo, the $\beta$ subunit of the hypoxia-inducible factors (HIF-1 and HIF-2) is constitutively expressed, while the $\alpha$ subunit is protected from degradation only under hypoxic condition, ${ }^{36}$ and HIF- $1 \alpha$ expression progressively increases from normal breast tissue to invasive carcinoma. Moreover, HIF-1 $\alpha$ expression is lower in well-differentiated than in poorly differentiated lesions, and it is associated with increased expression of the VEGF. ${ }^{37}$ The VEGF appears to be the most important growth factor involved in the regulation of the angiogenic process, and the intratumoral level of VEGF is considered a strong prognostic marker in patients with BC. ${ }^{38}$ The interaction between the VEGF and its receptors results in an autocrine loop that seems to promote the growth and metastasis of BC. ${ }^{39}$ Furthermore, the VEGFR-2, the most relevant endothelial cell surface receptor of the VEGF, is commonly overexpressed in $\mathrm{BC}{ }^{35}$ For these reasons, targeting the VEGFR-2 is a promising therapeutic approach in $\mathrm{MBC}$ patients, and ramucirumab is the only recombinant human monoclonal antibody that prevents the binding of the VEGF ligand to VEGFR-2 to be tested in MBC patients. ${ }^{40}$

Seven Asian women diagnosed with MBC were enrolled in a small Phase I study to receive docetaxel at a dose of $75 \mathrm{mg} / \mathrm{m}^{2}$ every 3 weeks in combination with ramucirumab administered as an intravenous infusion at a dose of $10 \mathrm{mg} / \mathrm{kg}$ every 3 weeks. ${ }^{41}$ Overall, four patients experienced a serious AE during the study treatment, with two patients experiencing a DLT. The decision to combine ramucirumab with docetaxel was based on the translational and clinical data available. In an in vivo BC model, treatment with a VEGF antibody plus docetaxel produced a more potent blockade of angiogenesis in the mice with VEGF-transfected cells compared with docetaxel alone, overcoming acquired resistance to docetaxel. ${ }^{42}$ In line with this result, the placebo-controlled Avastin and Docetaxel in Metastatic Breast Cancer (AVADO) trial demonstrated a longer PFS in human epidermal growth factor receptor 2-negative $\mathrm{MBC}$ patients treated with first-line 
bevacizumab in combination with docetaxel compared with patients treated with docetaxel single agent ( 8.1 months with docetaxel single agent vs 9.0 months with docetaxel plus bevacizumab $15 \mathrm{mg} / \mathrm{kg} ; P=0.045$ ). However, no significant improvement in OS was observed. ${ }^{43}$

At the 2014 ASCO Annual Meeting, the results of a Phase II trial comparing eribulin mesylate $1.4 \mathrm{mg} / \mathrm{m}^{2}$ on day 1 and day 8 every 3 weeks alone or in combination with ramucirumab $10 \mathrm{mg} / \mathrm{kg}$ on day 1 every 3 weeks in MBC patients pretreated with anthracycline and taxane were presented. ${ }^{44}$ The study did not meet its primary end point with a PFS of 4.4 months in the ramucirumab arm versus 4.1 months in the nonramucirumab arm $(P=0.4)$ and an OS of 13.5 months in the ramucirumab arm versus 11.5 months in the nonramucirumab arm $(P=0.4)$. No difference was observed either in ORR or in median duration of response. Moreover, higher rates of any grade fatigue ( $64 \%$ vs $57 \%$ ), headache ( $39 \%$ vs $15 \%$ ), diarrhea ( $25 \%$ vs $15 \%)$, bleeding ( $4.6 \%$ vs $18.8 \%$ ), hypertension ( $1.5 \%$ vs $13.0 \%$ ), and congestive heart failure ( $0 \%$ vs $1.4 \%$ ) were observed with the addition of the antiangiogenic drug.

Recently, an open-label, Phase II trial started to randomize patients with locally advanced or MBC who have been previously treated with anthracycline and taxane therapy to receive ramucirumab or icrucumab (IMC-18F1, a human antibody targeting VEGFR-1) administered every 3 weeks in combination with oral capecitabine administered twice a day on days $1-14$ every 3 weeks. ${ }^{45}$ Approximately 150 patients will be randomized and stratified by triple-negative receptor status and prior antiangiogenic therapy. The study is expected to complete the accrual of patients by the end of 2014; no preliminary results are available yet.

The results from the interim analysis of the Phase III trial combining docetaxel plus ramucirumab in MBC patients were initially presented at the 2013 San Antonio Breast Cancer Symposium. The ROSE/TRIO- 12 trial $^{46}$ randomized at a 2:1 ratio 1,144 human epidermal growth factor receptor 2-negative MBC patients to receive docetaxel $75 \mathrm{mg} / \mathrm{m}^{2}$ alone or in combination with ramucirumab $10 \mathrm{mg} / \mathrm{kg}$ every 3 weeks. After a median follow-up of 16.2 months, the addition of ramucirumab showed no significant advantage in either PFS or OS. The median PFS as assessed by the investigators was 9.5 months in the ramucirumab arm and 8.2 months in the nonramucirumab arm (HR 0.88; 95\% CI $0.95-1.01 ; P=0.077)$. Moreover, in an analysis stratified according to age, prior taxane therapy, visceral metastasis, hormone receptor status, and geographical region, no advantage to ramucirumab was found for any subgroup. The OS observed in an interim analysis showed no difference between arms, with a median OS of 27.3 months in the ramucirumab arm and 27.2 months in the nonramucirumab arm $(\mathrm{HR}=1.01$; $P=0.915$ ).

No difference was observed either in terms of antitumor activity with an ORR of $44.7 \%$ in the ramucirumab arm and $37.9 \%$ in the nonramucirumab arm $(P=0.027)$; the DCR was $86.4 \%$ versus $81.3 \%(P=0.022)$, respectively. The only significant difference observed between the investigational and the control arm was the median time to progression $(9.7$ months vs 8.2 months, respectively, HR $0.78 ; P=0.034$ ).

In line with the previously reported Phase II study, treatment-related AEs occurred at a higher rate in the ramucirumab arm compared with the nonramucirumab arm. In particular, patients treated with the antiangiogenic drug experienced more frequently, fatigue $(68.4 \%$ vs $66.0 \%)$, weight loss $(21.9 \%$ vs $10.5 \%)$, neutropenia ( $17.6 \%$ vs $16 \%)$, febrile neutropenia ( $8.1 \%$ vs $4.2 \%$ ), hypertension ( $27.0 \%$ vs $11.5 \%)$, bleeding ( $48.0 \%$ vs $22.3 \%$ ), epistaxis ( $39.9 \%$ vs $16.8 \%$ ), and stomatitis $(50.7 \%$ vs $30.6 \%)$.

So far, no antiangiogenic strategy has proved to improve OS in MBC. Hopefully, new translational trials such as the MERiDiAN study ${ }^{47}$ will outline biomarkers that can identify patients who will benefit the most from VEGF-targeted agents.

\section{Future directions and conclusion}

Ramucirumab alone or in combination with chemotherapy has demonstrated significant improvement in OS and PFS in pretreated patients with advanced gastric cancer or NSCLC. These results are relevant, especially in gastric cancer, because second-line treatment in both tumor types provides limited advantage in terms of OS, arising doubts and concerns about the cost efficacy profile of the drug. However, the scenario in gastric cancer compared with NSCLC is completely different. In gastric cancer there was no standard second-line treatment approved before ramucirumab, and despite the failure of bevacizumab to improve OS, the VEGF pathway seems to be relevant and to play a key role in this disease. The benefit of ramucirumab translated into a mortality RR of $22 \%$ with a notable $52 \%$ reduction in the risk of progression. The REGARD results are further corroborated by the positive results from the RAINBOW trial where ramucirumab was combined with paclitaxel in second-line treatment and compared with paclitaxel alone. Ramucirumab is a landmark treatment of gastric cancer, but the early disease progression in most patients suggests that the benefit to select patients who could benefit from this drug is an important issue to 
consider for the future development and use of ramucirumab in this disease.

In advanced NSCLC, three drugs are currently approved in second-line treatment, and ramucirumab has shown to provide a mortality RR of $14 \%$ and a $24 \%$ reduction in the risk of progression. These results, however, are inferior and less exciting compared with those achieved in gastric cancer trials. With only 1.4 months of difference in OS in favor of ramucirumab for patients with advanced NSCLC, the use of ramucirumab might be questioned also in light of the recent ASCO statement defining which outcomes should be considered as clinically meaningful. ${ }^{48}$ According to this statement, the survival benefit for NSCLC should be 2.5-4 months and 3-4 months for PFS in order to be considered clinically relevant. The REVEL results did not meet this criterion, which, however, is to be expected for first-line treatments and not for second-line therapies; therefore, it is reasonable to suggest that for NSCLC secondline treatments we should lower the threshold. Moreover, the 1.4 months of benefit in OS for ramucirumab could suggest that the improved PFS of 1.5 months did not just extend time on treatment but translated into prolonged survival. Another issue in favor of ramucirumab is the potential use in all-comers without histotype selection, because patients with squamous cell carcinoma seemed to have similar benefit from ramucirumab compared with the nonsquamous group. For this reason, ramucirumab could be a good option for pretreated patients with squamous cell carcinoma, potentially being the only antiangiogenic agent for this histotype, as bevacizumab is registered for nonsquamous cell carcinoma, and nintedanib has positive results in OS in adenocarcinoma only. ${ }^{49}$ Nevertheless, in the era of targeted agents, a biomarker-selected population should be considered the standard approach. But as regards ramucirumab, we can only remain hopeful that the identification of a specific biomarker might help select patients who will respond. Clearly, an adequate selection would be paramount for achieving better results and containing the expenses for this drug, which has a prohibitive cost (\$7,140 per infusion) and which would be used to treat tumors with high incidence like the NSCLCs.

\section{Disclosure}

Giuseppe Aprile was compensated as speaker for Eli-Lilly. Also, he was involved as principal investigator in the REGARD trial. Francesco Grossi was compensated as speaker for Eli-Lilly and received fees for participating in sponsored meetings and advisory boards. Erica Rijavec,
Caterina Fontanella, and Karim Rihawi have nothing to disclose.

\section{References}

1. Hicklin DJ, Ellis LM. Role of the vascular endothelial growth factor pathway in tumor growth and angiogenesis. J Clin Oncol. 2005;23(5): 1011-1027.

2. Kerbel RS. Tumor angiogenesis. N Engl J Med. 2008;358(19): 2039-2049.

3. Limaverde-Sousa G, Sternberg C, Ferreira CG. Antiangiogenesis beyond VEGF inhibition: a journey from antiangiogenic singletarget to broad-spectrum agents. Cancer Treat Rev. 2014;40(4): 548-557.

4. Schneider BP, Shen F, Miller KD. Pharmacogenetic biomarkers for the prediction of response to antiangiogenic treatment. Lancet Oncol. 2012;13(10):e427-e436.

5. Lambrechts D, Lenz HJ, de Haas S, Carmeliet P, Scherer SJ. Markers of response for the antiangiogenic agent bevacizumab. J Clin Oncol. 2013;31(9):1219-1230.

6. Tocchetti CG, Gallucci G, Coppola C, et al. The emerging issue of cardiac dysfunction induced by antineoplastic angiogenesis inhibitors. Eur J Heart Fail. 2013;15(5):482-489.

7. Ferrara N, Gerber HP, LeCouter J. The biology of VEGF and its receptors. Nat Med. 2003;9(6):669-676.

8. Youssoufian H, Hicklin DJ, Rowinsky EK. Review: monoclonal antibodies to the vascular endothelial growth factor receptor-2 in cancer therapy. Clin Cancer Res. 2007;13(18 Pt 2):5544s-5548s.

9. Aprile G, Bonotto M, Ongaro E, at al. Critical appraisal of ramucirumab (IMC-1121B) for cancer treatment: from benchside to clinical use. Drugs. 2013;73:2003-2015.

10. Lu D, Jimenez X, Zhang H, et al. Selection of high affinity human neutralizing antibodies to VEGFR2 from a large antibody phage display library for antiangiogenesis therapy. Int J Cancer. 2002;97(3):393-399.

11. Miao HQ, Hu K, Jimenez X, et al. Potent neutralization of VEGF biological activities with a fully human antibody Fab fragment directed against VEGF receptor 2. Biochem Biophys Res Commun. 2006;345(1): 438-445.

12. Poone RM, Vaidya A. Ramucirumab: first global approval. Drugs. 2014;74(9):1047-1058.

13. Prewett M, Huber J, Li Y, et al. Antivascular endothelial growth factor receptor (fetal liver kinase 1) monoclonal antibody inhibits tumor angiogenesis and growth of several mouse and human tumors. Cancer Res. 1999;59:5209-5218.

14. Bruns CJ, Liu W, Davis DW, et al. Vascular endothelial growth factor is an in vivo survival factor for tumor endothelium in a murine model of colorectal carcinoma liver metastases. Cancer. 2000;89:488-499.

15. Zhu Z, Hattori K, Zhang H, et al. Inhibition of human leukemia in an animal model with human antibodies directed against vascular endothelial growth facto receptor 2. Correlation between antibody affinity and biological activity. Leukemia. 2003;17:604-611.

16. Clarke JM, Hurwitz HI. Targeted inhibition of VEGF receptor 2: an update on ramucirumab. Expert Opin Biol Ther. 2013;13(8):1187-1196.

17. Spratlin JL, Cohen RB, Eadens M, et al. Phase I pharmacologic and biologic study of ramucirumab (IMC-1121B), a fully human immunoglobulin G1 monoclonal antibody targeting the vascular endothelial growth factor receptor-2. J Clin Oncol. 2010;28:780-787.

18. Fuchs CS, Tomasek J, Yong CJ, et al. Ramucirumab monotherapy for previously treated advanced gastric or gastro-oesophageal junction adenocarcinoma (REGARD): an international, randomised, multicentre, placebo-controlled, phase 3 trial. Lancet. 2014;383(9911):31-39.

19. Safran H, Tomasek J, Yong CJ, et al. REGARD: a phase 3, randomized, double-blind trial of ramucirumab and best supportive care versus placebo and BSC in the treatment of metastatic gastric or gastroesophageal junction adenocarcinoma following disease progression on first-line platinum- and/or fluoropyrimidine-containing combination therapy: age subgroup analysis. ASCO Annual Meeting; 2014. 
20. Wilke H, Van Cutsem E, Oh SC, et al. RAINBOW: a global, phase III, randomized, double-blind study of ramucirumab plus paclitaxel versus placebo plus paclitaxel in the treatment of metastatic gastroesophageal junction (GEJ) and gastric adenocarcinoma following disease progression on first-line platinum- and fluoropyrimidine-containing combination therapy rainbow IMCL CP12-0922 (I4T-IE-JVBE). Gastrointestinal Cancers Symposium; 2014.

21. Wilke H, Van Cutsem E, Oh SC, et al. RAINBOW: a global, phase 3, randomized, double-blind study of ramucirumab plus paclitaxel versus placebo plus paclitaxel in the treatment of metastatic gastric adenocarcinoma following disease progression on first-line platinumand fluoropyrimidine-containing combination therapy: results of a multivariate Cox regression analysis adjusting for prognostic factors. ASCO Annual Meeting; 2014:abstract 4076.

22. Van Cutsem E, Clingan P, Ananda S, et al. RAINBOW: a global, phase 3, double-blind study of ramucirumab (RAM) plus paclitaxel (PTX) versus placebo $(\mathrm{PL})+\mathrm{PTX}$ in the treatment of advanced gastric and gastroesophageal junction (GEJ) adenocarcinoma following disease progression on first-line platinum- and fluoropyrimidine-containing combination therapy: a subgroup analysis of Western population. ASCO Annual Meeting; 2014.

23. Al-Batran SH, Van Cutsem E, Oh SC, et al. RAINBOW: a global, phase III, randomized, double-blind study of ramucirumab plus paclitaxel versus placebo plus paclitaxel in patients with previously treated gastric or gastroesophageal junction (GEJ) adenocarcinoma - quality-of-life (QoL) results. ASCO Annual Meeting; 2014:abstract 4058.

24. Yoon HH, Bendell JC, Fadi SB, et al. Ramucirumab (RAM) plus FOLFOX as front-line therapy ( $\mathrm{Rx})$ for advanced gastric or esophageal adenocarcinoma (GE-AC): randomized, double-blind, multicenter phase 2 trial. Presented at ASCO 2014 Annual Meeting. J Clin Oncol. 2014;325:abstract 4004.

25. Eli Lilly and Company. A study of ramucirumab (IMC-1121B) drug product (DP) and best supportive care (BSC) versus placebo and BSC as 2nd-line treatment in patients with hepatocellular carcinoma after 1st-line therapy with sorafenib (REACH). http://www.clinicaltrials. gov/ct2/show/NCT01140347?term=NCT01140347\&rank=1. NLM identifier: NCT01140347. Accessed September 25, 2014.

26. Eli Lilly and Company. A study in second line metastatic colorectal cancer. http://www.clinicaltrials.gov/ct2/show/NCT01183780?term= NCT01183780\&rank=1. NLM identifier: NCT01183780. Accessed September 25, 2014.

27. Grothey A, Tabernero J, Rougier P, et al. A randomized, doubleblind, phase $(\mathrm{Ph})$ III study of the irinotecan-based chemotherapy FOLFIRI plus ramucirumab (RAM) or placebo (PL) in patients (pts) with metastatic colorectal carcinoma (mCRC) progressive during or following first-line therapy with bevacizumab (BEV), oxaliplatin (OXALI), and a fluoropyrimidine (FP) (RAISE) (NCT01183780). 2014 ASCO Annual Meeting. J Clin Oncol. 2012;30(Suppl):abstract TPS3634.

28. Zhu AX, Chau I, Blanc J-F, et al. A multicenter, randomized, doubleblind, phase III study of ramucirumab (IMC-1121B; RAM) and best supportive care (BSC) versus placebo (PBO) and BSC as second-line treatment in patients (pts) with hepatocellular carcinoma (HCC) following first-line therapy with sorafenib (SOR). 2012 ASCO Annual Meeting. J Clin Oncol. 2012;30(Suppl):abstract TPS4146. NCT01140347.

29. Camidge DR, Ballas MS, Dubey S, et al. A phase II, open-label study of ramucirumab (IMC-1121B), an IgG1 fully human monoclonal antibody (Mab) targeting VEGFR-2, in combination with paclitaxel and carboplatin as first-line therapy in patients (pts) with stage IIIb/IV non-small cell lung cancer (NSCLC). J Clin Oncol. 2010; 28(Suppl):7588.

30. Doebele RC, Spigel D, Tehfe M, et al. A phase 2 randomized openlabel study of ramucirumab (IMC 1121B; RAM) in combination with first-line platinum-based chemotherapy in patients (pts) with recurrent or advanced non-small cell lung cancer (NSCLC): final results from non-squamous (NSQ) pts. J Thorac Oncol. 2013;8:S290.
31. Garon EB, Cao D, Alexandris E, John WJ, Yurasov S, Perol M. A randomized, double-blind, phase III study of docetaxel and ramucirumab versus docetaxel and placebo in the treatment of stage IV non-small-cell lung cancer after disease progression after 1 previous platinum-based therapy (REVEL): treatment rationale and study design. Clin Lung Cancer. 2012;13:505-509.

32. Garon EB, Ciuleanu TE, Arrieta O, et al. Ramucirumab plus docetaxel versus placebo plus docetaxel for second-line treatment of stage IV non-small-cell lung cancer after disease progression on platinum-based therapy (REVEL): a multicentre, double-blind, randomized phase 3 trial. Lancet. 2014;384(9944):665-673.

33. Eli Lilly and Company. An open-label, multicenter, randomized, phase 2 study of a recombinant human anti-VEGFR-2 monoclonal antibody, IMC-1121B in combination with platinum-based chemotherapy versus platinum-based chemotherapy alone as first-line treatment of patients with recurrent or advanced non-small cell lung cancer (NSCLC). Available from: http://clinicaltrials.gov/show/NCT01160744. NLM identifier: NCT01160744. Accessed September 25, 2014.

34. Eli Lilly and Company. A randomized, double-blind, phase 2 study of docetaxel and ramucirumab versus docetaxel and placebo in the treatment of stage IV non-small cell lung cancer following disease progression after one prior platinum-based therapy. Available from: http:// clinicaltrials.gov/show/NCT01703091. NLM identifier: NCT01703091. Accessed September 25, 2014.

35. Aesoy R, Sanchez BC, Norum JH, Lewensohn R, Viktorsson K, Linderholm B. An autocrine VEGF/VEGFR2 and p38 signaling loop confers resistance to 4-hydroxytamoxifen in MCF-7 breast cancer cells. Mol Cancer Res. 2008;6:1630-1638.

36. Ghosh S, Sullivan CA, Zerkowski MP, et al. High levels of vascular endothelial growth factor and its receptors (VEGFR-1, VEGFR-2, neuropilin-1) are associated with worse outcome in breast cancer. Hum Pathol. 2008:39:1835-1843.

37. Skobe M, Rockwell P, Goldstein N, Vosseler S, Fusenig NE. Blocking angiogenesis results in tumor cell invasion suppression. Nat Med. 1997;3:1222-1227.

38. Konecny GE, Meng YG, Untch M, et al. Association between HER-2/ neu and vascular endothelial growth factor expression predicts clinical outcome in primary breast cancer patients. Clin Cancer Res. 2004;10: 1706-1716.

39. Price DJ, Miralem T, Jiang S, Steinberg R, Avraham H. Role of vascular endothelial growth factor in the stimulation of cellular invasion and signaling of breast cancer cells. Cell Growth Differ. 2001;12: 129-135.

40. O’Sullivan Coyne G, Burotto Pichun M. Clinical experience with ramucirumab: outcomes in breast cancer. Exp Opin Biol Ther. 2014;14(9): 1351-1360.

41. Eli Lilly and Company. A study of ramucirumab (IMC-1121B) in participants with breast cancer. Available from: http://clinicaltrials. gov/ct2/show/results/NCT01256567. NLM identifier: NCT01256567. Accessed September 25, 2014.

42. Hotchkiss KA, Ashton AW, Sparano J, et al. Inhibition of endothelial cell function by docetaxel (Taxotere). Proc Am Assoc Cancer Res. 2000;41:647.

43. Miles DW, Chan A, Dirix LY, et al. Phase III study of bevacizumab plus docetaxel compared with placebo plus docetaxel for the first-line treatment of human epidermal growth factor receptor 2-negative metastatic breast cancer. J Clin Oncol. 2010;28:3239-3247.

44. Yardley DA, Richards PD, Reeves JA, et al. Final results of a phase 2 study of ramucirumab (RAM) plus eribulin (E) versus $E$ in advanced metastatic breast cancer (MBC). J Clin Oncol. 2014;32(Suppl): abstract 1035 .

45. Eli Lilly and Company. Study of IMC- $18 \mathrm{~F} 1$ or ramucirumab DP in combination with capecitabine or capecitabine on previously treated breast cancer patients. Available from: http://clinicaltrials. gov/show/NCT01234402. NLM identifier: NCT01234402. Accessed September 25, 2014. 
46. Mackey JR, Ramos-Vazquez M, Lipatov O, et al. Primary results of ROSE/TRIO-12, a randomized placebo controlled phase III trial evaluating the addition of ramucirumab to first-line docetaxel chemotherapy in metastatic breast cancer. Available from: http://www.abstracts2view. com/sabcs13/view.php?nu=SABCS13L_238. Accessed September 25, 2014.

47. Miles DW, de Haas SL, Dirix LY, et al. Biomarker results from the AVADO phase 3 trial of first-line bevacizumab plus docetaxel for HER2-negative metastatic breast cancer. Br J Cancer. 2013;19;108(5): 1052-1060.
48. Ellis LM. American Society of Clinical Oncology perspective: raising the bar for clinical trials by defining clinically meaningful outcomes. $J$ Clin Oncol. 2014;32(12):1277-1280.

49. Reck M, Kaiser M, Mellemgaard A, et al. Docetaxel plus nintedanib versus docetaxel plus placebo in patients with previously treated non-small-cell lung cancer (LUME-Lung 1): a phase 3, double-blind, randomised controlled trial. Lancet Oncol. 2014;15(2): 143-155.

\section{Publish your work in this journal}

OncoTargets and Therapy is an international, peer-reviewed, open access journal focusing on the pathological basis of all cancers, potential targets for therapy and treatment protocols employed to improve the management of cancer patients. The journal also focuses on the impact of management programs and new therapeutic agents and protocols on

\section{Dovepress}

patient perspectives such as quality of life, adherence and satisfaction. The manuscript management system is completely online and includes a very quick and fair peer-review system, which is all easy to use. Visit http://www.dovepress.com/testimonials.php to read real quotes from published authors. 